\title{
Spatial domain image enhancement techniques for acute myeloid leukemia (M1,M4,M5,M7)
}

\author{
A.S.A.Salam ${ }^{1}$, M.N.M.Isa ${ }^{2}$, M.I.Ahmad ${ }^{3}$ \\ ${ }^{1,2}$ School of Microelectronic Engineering, Universiti Malaysia Perlis, Malaysia \\ ${ }^{3}$ School of Computer and Communication Engineering, Universiti Malaysia Perlis, Malaysia
}

\begin{tabular}{l}
\hline \hline Article Info \\
\hline Article history: \\
Received Sep 30, 2018 \\
Revised Nov 26, 2018 \\
Accepted Nov 5, 2018 \\
\hline Keywords: \\
AML \\
Contrast stretching \\
Image enhancement \\
Image sharpening \\
Image subtraction \\
Leukemia
\end{tabular}

\begin{abstract}
In this paper, several techniques of image enhancement spatial domain is elucidated and analyzed for the purpose of enhancing Acute Myeloid Leukemia (AML) subtype of M1, M4, M5 and M7. The techniques involved contrast stretching of greyscale images, image subtraction and image sharpening. The three methods compared with one another to achieve the highest PSNR value for the suitability technique of AML subtypes (M1, M4, M5 and M7). Firstly, subtypes images converted into grayscale. Then, each four images tested with contrast stretching techniques followed by image subtraction and image sharpening. The performances were evaluated based on Mean Square Error (MSE) and Peak Signal to Noise Ratio (PSNR). Due to its higher value obtained, image sharpening is a good enhancement techniques for Acute Myeloid Leukemia with $68.2083 \mathrm{~dB}$ and the lowest MSE achieved of 0.0103 .
\end{abstract}

Copyright (C) 2019 Institute of Advanced Engineering and Science. All rights reserved.

\section{Corresponding Author:}

Mohd Nazrin bin Md Isa,

School of Microelectronic Engineering,

Universiti Malaysia Perlis,

Pauh Putra Campus, 02600,Arau, Perlis, Malaysia.

Email: nazrin@unimap.edu.my

\section{INTRODUCTION}

In medicinal field, medical imaging is one of the controlling apparatus for having an insight on the pathological procedures. Images that includes computer tomography (CT), magnetic resonance imaging (MRI), ultrasound and X-ray are one of the focal bases for diacrisis of diseases. The main purposes of medical image processing is to diagnose medical images more efficiently and accurately. Typically, these images are affected by noise, blurriness and other bad qualities that interrupts the quality of the image.[1][2] Thus, image enhancement techniques can improve the visual appearance of medical images especially in detecting Leukemic cell.

\subsection{Leukemia}

Leukemia are bone marrow cancerous cell, which involves proliferation of white blood cells that disables its main function to fight bacteria more efficiently. The blast cell are grouped by affected blood cell type called lymphocytes and myelocytes.[3] The disease also categorized by its speed of illnesses. Acute means the cells spreads fast while chronic takes time growing but do worsens over the year. Thus, Leukemia are grouped by four main types which are acute lymphocytic leukemia $(A L L)$, chronic lymphocytic leukemia $(C L L)$, acute myelocytic leukemia $(A M L)$ and chronic myelocytic leukemia $(C M L)$. AML type leukemia usually detected until it has spread into other organs. The cells classified by a system known as French-American British (FAB), which categorized into eight subtypes as shown in Table 1. 
Table 1. French-American-British Classification [4]

\begin{tabular}{cl}
\hline FAB Subtypes & \multicolumn{1}{c}{ Name } \\
\hline M0 & Undifferentiated acute myeloblastic leukemia \\
M1 & Acute myeloblastic leukemia with minimal maturation \\
M2 & Acute myeloblastic leukemia with maturation \\
M3 & Acute promyelocytic leukemia (APL) \\
M4 & Acute myelomonocytic leukemia \\
M4 eos & Acute myelomonocytic leukemia with eosinophilia \\
M5 & Acute monocytic leukemia \\
M6 & Acute erythroid leukemia \\
M7 & Acute megakaryoblastic leukemia \\
\hline
\end{tabular}

In Malaysia, Myeloid type states the highest amount for both male and female as depicted in Figure 1 and Figure 2.

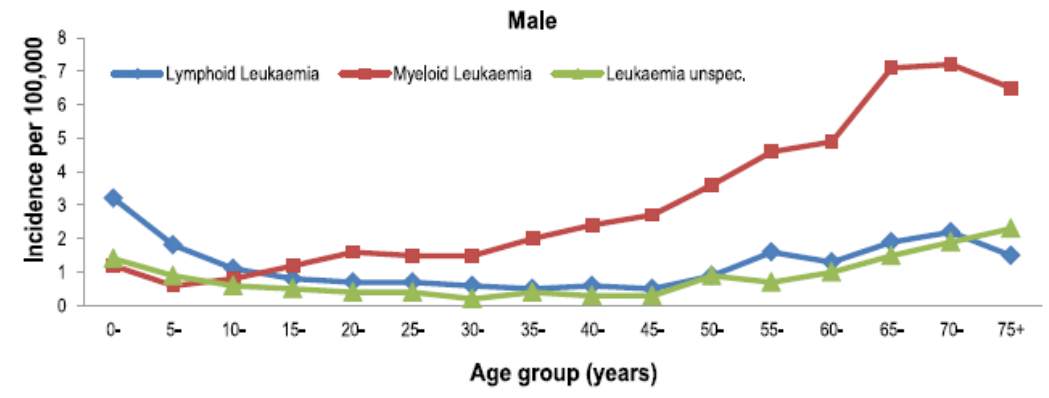

Figure 1. Leukemia types age-specific incidence rate, males, Malaysia, 2007-2011 [5]

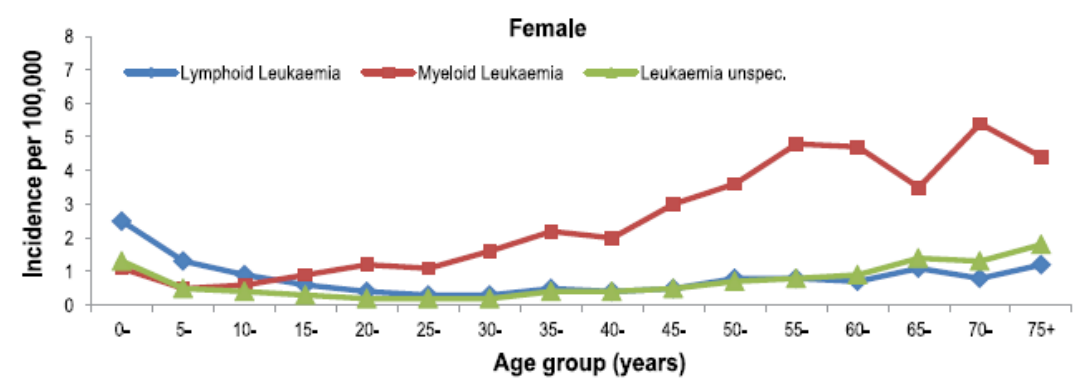

Figure 2. Leukemia types age-specific incidence rate, females, Malaysia, 2007-2011

Based on the graph, it shows that Myeloid in Malaysia is severe. Hence, alternative early diagnostic to enhance current hospitality are much needed. This is where medical image processing becomes useful for diagnosing early detection of AML subtypes.

\subsection{Image Enhancement}

In image processing, image enhancement is capable of improving the image's contrast for making various features to be easily recognized.[6].The role is vital in enhancing quality of medical images such as AML because the image's quality depends on the exposure of the microscope and staining process[7]. Enhancement techniques are divided into two broad categories:

a) Spatial domain methods

b) Frequency domain methods

Spatial domain operates on the pixel whilst frequency domain are computed in Fourier transform in order to modify the frequency content of the image so that edges and other subtle information can be enhanced [8], [9]. This paper solely focuses on spatial domain methods. The term itself works in the given space (the image). This means that the procedure works directly on pixels as shown on Figure 3.

Spatial domain image enhancement techniques for acute myeloid leukemia (M1,M4,M5,M7) (A.S.A.Salam ) 


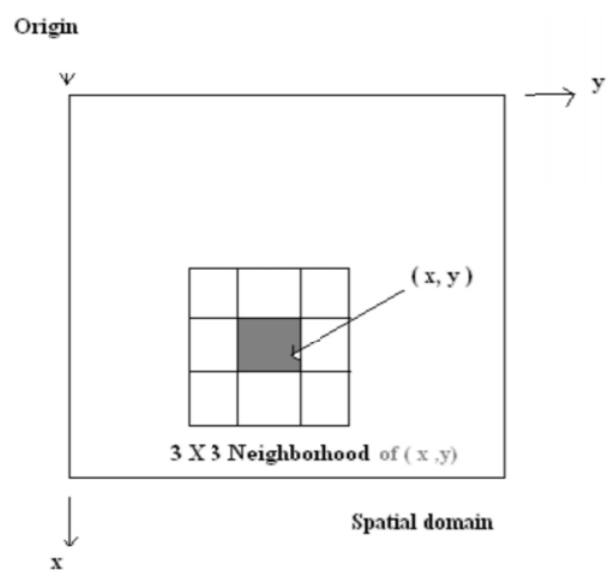

Figure 3. Spatial domain of an image [9]

Spatial domain expression are denoted by the expression,

$$
g(x, y)=T[f(x, y)]
$$

Where $\mathrm{g}(\mathrm{x}, \mathrm{y})=$ processed image

$\mathrm{f}(\mathrm{x}, \mathrm{y})=$ input image

$\mathrm{T}=$ operator on $f$ defined over some neighborhood of $(\mathrm{x}, \mathrm{y})$ if using $(x, y)$

There are many techniques that can be involved in spatial domain enhancement such as contrast stretching, image subtraction, sharpening, histogram equalization, log transformations etc... Author [8] conducted a survey on spatial domain techniques which involved point processing, Histogram stretching, Histogram equalization, sharpening filtering and many more. Based on the review, the effectiveness of each techniques becomes more effective when combined more than one method. Whilst [9] works on comparing spatial and frequency enhancement and obtained result shows that spatial domain are apt for small kernel since Fourier transform takes time. On the other hand, [10] implemented image sharpening and smoothing by filters on the particular image. The input image sharpened by using weighted kernel of different values and gives sharper image with the boundary edge information. The analyzed results performed by MSE and PSNR. Research by[6] surveys variety of contrast enhancement techniques like Histogram equalization (HE), Contrast limited Adaptive Histogram Equalization (CLAHE), Morphological enhancement on a single scale and Multiscale Morphological enhancement. Compared result of these techniques onto a synthetic image of kidney and brain shows that the Multiscale morphological approach obtained respectable results as to the results achieved with other ultramodern techniques. Moreover, [11] improves image quality by analyses contrast enhancement, sharpening and noise reduction of the dataset BSDS300 Berkely. Quantitative performance involves MSE, PSNR and SSIM, which proves that image sharpening provides decent results due to no change of information or pixel as it is close to the information of the original image.

\section{RESEARCH METHOD}

This section explains the methodology of this paper. Firstly, apply input image of M1 then, the RGB image converted into greyscale. Next, implement contrast stretching on the given image. To find the performance, calculate MSE onto M1 followed by PSNR. These steps are continuous until all input images tested with contrast stretching techniques. After that, implement Image subtraction techniques onto the four images followed by Image sharpening. Finally, for each techniques, calculate the mean value of the four images as so to find which of the three enhancement techniques are apt for enhancing M1, M4, M5 and M7. Flow chart on Figure 4 depicts clearly on the explained methods.

Indonesian J Elec Eng \& Comp Sci, Vol. 14, No. 1, April 2019 : 250 - 257 


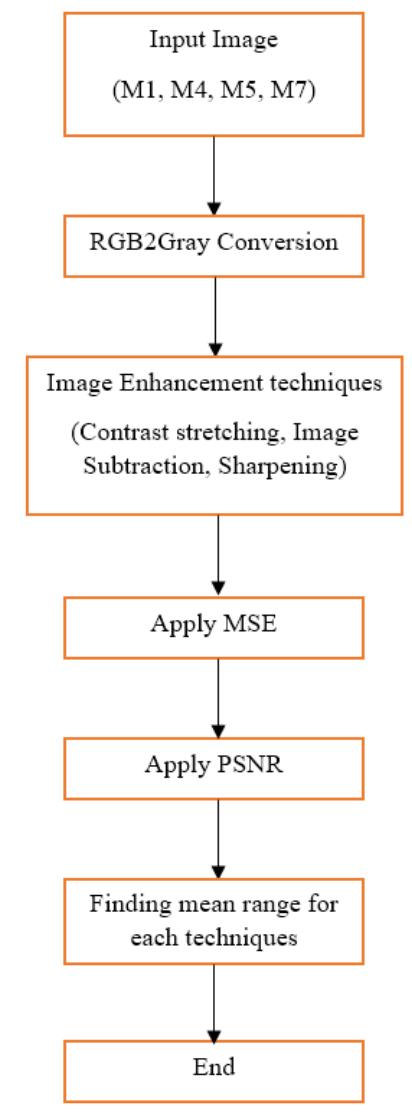

Figure 4. Overall Flowchart of the methods

Input images of M1, M4, M5 and M7 inserted according to Figure 5.

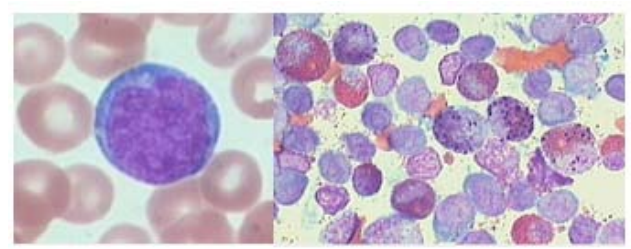

(a)

(b)

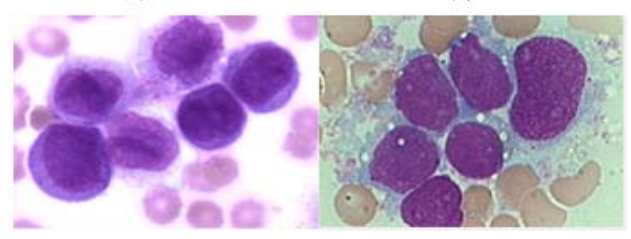

(c)

(d)

Figure 5. (a) RGB M1 (b) RGB M4 (c) RGB M5 (d) RGB M7

The RGB images convert into greyscale(Figure 6) to reduce dimension of image[12]. Also, processing becomes flexible when a single intensity value of each pixel is specified [13]. 


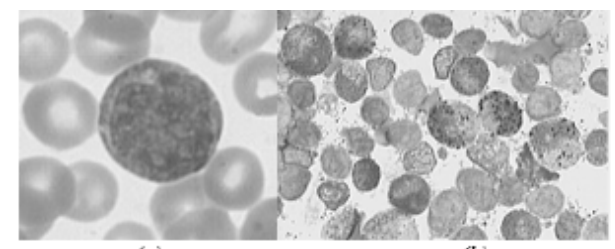

(a)

(b)

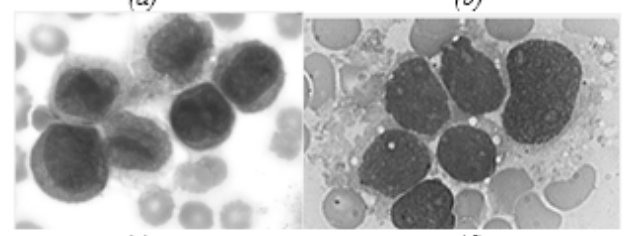

(c)

(d)

Figure 6. (a) Greyscale M1 (b) Greyscale M4 (c) Greyscale M5 (d) Greyscale M7

Contrast stretching attempts to improve the contrast of the image by stretching range of the intensity values. It changes the distribution and range of digital numbers assigned to each pixel in an image. In medical imaging, contrast stretching plays an important role for quality enhancement[14]. Figure 7 illustrates the results from stretching.

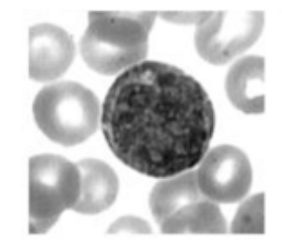

(a)

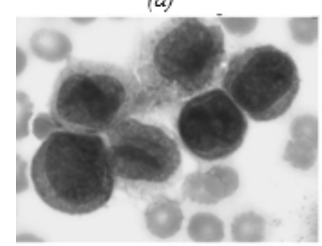

(c)

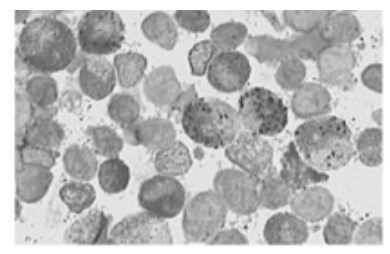

(b)

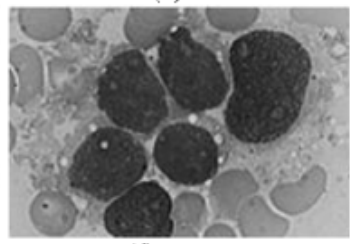

(d)

Figure 7. (a) Contrast M1 (b) Contrast M4 (c) Contrast M5 (d) Contrast M7

Image subtraction is one of the popular machine vision technique for extracting foreground objects in an image[15].It is obtained by computing the difference between all pairs of corresponding pixels from $f$ and $\mathrm{h}$

$$
G(x, y)=f(x, y)-h(x, y)
$$

Where $G(x, y)=$ Image Subtraction

$f(x, y)=$ Image background

$\mathrm{h}(\mathrm{x}, \mathrm{y})=$ Image foreground 


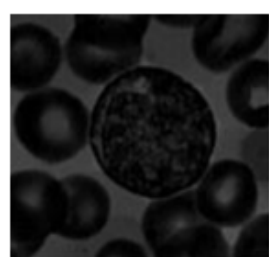

(a)

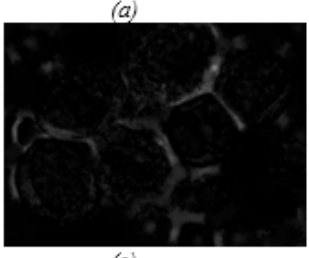

(c)
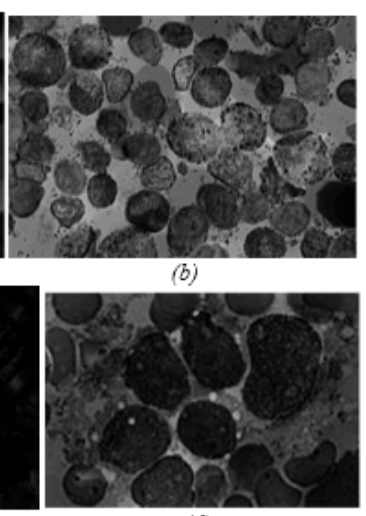

(d)

Figure 8. (a) Subtraction M1 (b) Subtraction M4 (c) Subtraction M5 (d) Subtraction M7

The aim of sharpening in image processing is to enhance the details of an image or even line structures. The techniques designed to increase the high frequency aspects of the image. In details, sharpening contains of adding signal that is proportional to a high-pass filtered version of the original image.[11].

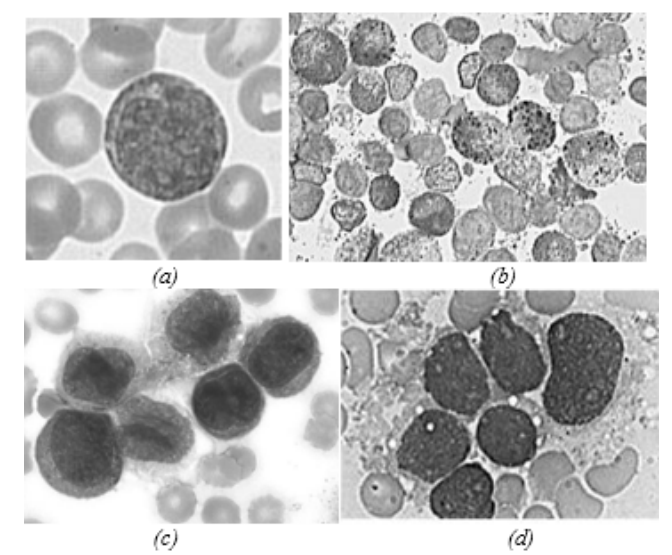

Figure 8. (a) Sharpening M1 (b) Sharpening M4 (c) Sharpening M5 (d) Sharpening M7

The images were analysed by using the Mean Squared Error(Mse) and Peeak Signal to Noise Ratio(PSNR). MSE represents the cumulative squared error between compressed image and original image [2]. The lower the MSE value, the lower the error rate.

$$
M S E=\frac{\sum_{M, N}\left[I_{1}(m, n)-I_{2}(m, n)\right]^{2}}{M \times N}
$$

Where $\mathrm{I}_{2}(\mathrm{~m}, \mathrm{n})=$ original image

$\mathrm{I}_{1}(\mathrm{~m}, \mathrm{n})=$ output image

$\mathrm{M}, \mathrm{N}$ is the size of image

PSNR measures the peak error. It computes the peak signal-to-noise ratio in decibels between two images. The higher the PSNR value, the better the quality of the image.

$$
P S N R=10 \log _{10}\left(\frac{R^{2}}{M S E}\right)
$$


Where $\mathrm{R}^{2}=2 \mathrm{n}^{\mathrm{n}}-1$ and $\mathrm{n}$ represents number of bits. In representing the pixel of the image.

\section{RESULTS AND ANALYSIS} and Table 4 .

After the MSE and PSNR have calculated, the results are tabulated as shown on Table 2, Table 3

Table 2. Results on Contrast Stretching

\begin{tabular}{ccc}
\hline Subtypes & MSE & $\begin{array}{c}\text { PSNR } \\
\text { dB }\end{array}$ \\
\hline M1 & 0.0240 & 64.3290 \\
M4 & 0.0006, & 70.1579 \\
M5 & 0.0256 & 64.0474 \\
M7 & 0.0240 & 64.3246 \\
\hline
\end{tabular}

Table 3.Results on Image Subtraction

\begin{tabular}{ccc} 
Subtypes & MSE & $\begin{array}{c}\text { PSNR } \\
\text { dB }\end{array}$ \\
\hline M1 & 0.3831 & 52.2981 \\
M4 & 0.1879 & 55.3914 \\
M5 & 0.5163 & 51.0017 \\
M7 & 0.1894 & 55.3580 \\
\hline
\end{tabular}

Table 4. Results on Image Sharpening

\begin{tabular}{ccc} 
Subtypes & MSE & $\begin{array}{c}\text { PSNR } \\
\text { dB }\end{array}$ \\
\hline M1 & 0.0060 & 70.3261 \\
M4 & 0.0133 & 66.9005 \\
M5 & 0.0096 & 68.2993 \\
M7 & 0.0121 & 67.3073 \\
\hline
\end{tabular}

Since the aim of this paper is to analyze and select which techniques is apt in enhancing AML subtype, a mean value of each techniques are calculated. To find the mean, total value of MSE and PSNR are divided over the number of subtypes. As shown on equation 4.

$$
\text { mean }=\frac{M 1 \oplus M 4 \oplus M 5 \oplus M 7}{4}
$$

Table 5. Mean values for Image Enhancement Techniques

\begin{tabular}{ccc} 
Enhancement Techniques & MSE & $\begin{array}{c}\text { PSNR } \\
\mathrm{dB}\end{array}$ \\
\hline Contrast Enhancement & 0.0186 & 65.7147 \\
Image Subtraction & 0.3192 & 53.5123 \\
Image Sharpening & 0.0103 & 68.2083 \\
\hline
\end{tabular}

As shown on Table 5, the results of mean values have tabulated. According to the result, Image sharpening achieved the highest PSNR of $68.2083 \mathrm{~dB}$. Hence, it shows good quality for enhancing AML subtypes M1, M4, M5, and M7. The technique agreed with [11] as the author also achieved image sharpening as a good result compared to other techniques.

\section{CONCLUSION}

Concisely, this paper is about experimenting on various image enhancement techniques such as contrast stretching, image subtraction and image sharpening. These techniques tested on AML subtype M1, M4, M5 and M7. Enhancing medical images is essential as it helps to improve the quality of the image. Performance analysis such as MSE and PSNR helps to analyze the tested techniques. Since a high PSNR value means a better quality of the particular image, Image sharpening shows good quality out of the other two techniques with a value of $68.2083 \mathrm{~dB}$. Future work is to test the performance of these images with other image quality measurement such as SSIM, F1 score or even profiling time performance.

\section{REFERENCES}

[1] M. Yasmin and M. Sharif, "Brain Image Enhancement-A Survey,” no. January, 2012.

Indonesian J Elec Eng \& Comp Sci, Vol. 14, No. 1, April 2019 : 250 - 257 
[2] M. Shanthi and M. Renuga, "Performance Analysis of Image Enhancement Techniques for kidney Image," Int. J. Adv. Res. Electr. Electron. Instrum. Eng., vol. 5, no. 5, pp. 3517-3522, 2016.

[3] A. S. Abdul Salam, M. N. Md. Isa, M. I. Ahmad, and R. Che Ismail, "Comparison of edge detection techniques for M7 subtype Leukemic cell in terms of noise filters and threshold value," EPJ Web Conf., vol. 162, 2017.

[4] a Carroll et al., "The $\mathrm{t}(1 ; 22)(\mathrm{p} 13 ; \mathrm{q} 13)$ is nonrandom and restricted to infants with acute megakaryoblastic leukemia: a Pediatric Oncology Group Study.," Blood, vol. 78, no. 3, pp. 748-752, 1991.

[5] A.. et. a. M, "Moh/p/ikn/01.16 (ar)," Putrajaya, Malaysia.

[6] S. K. Ritika, "Contrast Enhancement Techniques for Images - A Visual Analysis," Int. J. Comput. Appl., vol. 64, no. 17, pp. 20-25, 2013.

[7] N. Hazwani, A. Halim, M. Y. Mashor, and R. Hassan, "Automatic Blasts Counting for Acute Leukemia Based on Blood," vol. 2, no. 4, pp. 971-976, 2011.

[8] S. S. Negi and B. Gupta, "Survey of Various Image Enhancement," Intermational J. Comput. Appl., pp. 22-30, 2014.

[9] A. Sarah, "Image Enhancement -Spatial vs. Frequency Domain Filters," 2016.

[10] K. S. Praveen, K. P. Babu, and M. Sreenivasulu, "Implementation of Image Sharpening and," Int. Sci. Eng. Appl. Sci., vol. 2, no. 1, pp. 7-14, 2016.

[11] A. Nevriyanto and A. H. Equalization, "Enhancement, and Standard Median Filter ( Noise Removal ) with PixelBased and Human Visual System-Based Measurements," in International Conference on Electrical Engineering and Computer Science (ICECOS), 2017, vol. 1, no. 1, pp. 114-119.

[12] H. Vaghela, H. Modi, M. Pandya, and M. Potdar, "Leukemia Detection using Digital Image Processing Techniques," Int. J. Appl. Inf. Syst., vol. 10, no. 1, pp. 43-51, 2015.

[13] K. Raghul, A. S. Raj, and P. U. Ilavarasi, "Acute Lymphocytic Leukemia Detection by Image Processing Using Matlab," vol. 24, pp. 263-267, 2016.

[14] J. Kaur and A. Choudhary, "Comparison of Several Contrast Stretching Techniques on Acute Leukemia Images," Int. J. Eng. Innov. Technol., vol. 2, no. 1, pp. 332-335, 2012.

[15] L. Venetsky, R. Boczar, and R., "Optimization of background subtraction for image enhancement," Proc. SPIE Defense, Secur. Sens., no. May, pp. 875102-875102-13, 2013.

\section{BIOGRAPHIES OF AUTHORS}

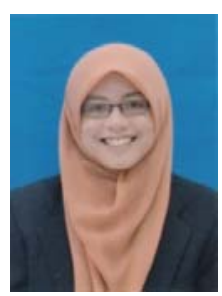

Ms. Afifah Salmi Abdul Salam is a Master by Research student in Universiti Malaysia Perlis under the School of Microelectronics. Her field of interest is Medical Image processing specifically in Acute Myeloid Leukemia detection. She graduated from Universiti Malaysia Terengganu, in 2016 with a Bachelor Degree of Applied Science (Physics, Electronic and Instrumentation).

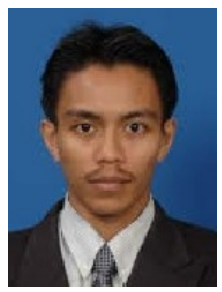

Dr. Mohd Nazrin Md Isa is a senior lecturer in the School of Microelectronic Engineering at Universiti Malaysia Perlis (UniMAP). Currently, he is a member of Integrated Circuits and Systems Design (ICASe) group. His research interests include reconfigurable architectures, bioinformatics and computational biology, field programmable gate array (FPGA) and ASIC design. He graduated his doctorate study from the University of Edinburgh, Scotland, UK in 2013. His PhD thesis entitled "High Performance Reconfigurable Architectures for Biological Sequence Alignments"

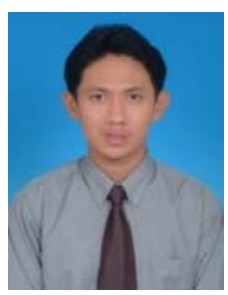

Dr Muhammad Imran Ahmad received his $\mathrm{PhD}$ in Computer Engineering from Newcastle University, United Kingdom in 2014. Currently he is a senior lecturer at School of Computer and Communication Engineering, Universiti Malaysia Perlis. His research interests include biometric, signal analysis and image processing. 